\title{
List of Members
}

The Historic Brass Society would like to thank its members, without whom neither it nor its Newsletter and Journal could exist. We are compiling a Membership Directory, which will be sent free of charge to all members. If you do not want your address and telephone number listed, please let us know before June 1, 1990.

Jon Michael Allsen
Eric Anderson
Mark Anderson
Ronald Anderson
Steve Anderson
Winthrop Armour
Thomas Axworthy
George T. Bachmann
Bad Sackingen
Trompeten Museum
Edward Bahr
Bob Barclay
Bruce Barrie
Peter Barton
Michael Bassichis
Ronald Bell
Frederick Benkovic
Philip Benson
Donald Beyer
Thomas E. Binkley
Martha Bixler
J.F. Bloemendaal
Anne Bonn
Stanley Boorman
Jon Borowicz
Ronald Borror
Kent Brandberry
Alex Brown
Ralph Bryant
M. Wilfried Buck
Ernst Buser
David Bushouse
Todd Caine
Frank Campos
Jackie T. Carter
Stewart Carter
Stephen Chenette
Gaetan Chenier
Trention

Gilbert Cline
Richard Clymer
Sandra Coffin
Michael Collver
Charles Conrad
John Cook
Stephen Coyne
Robert Cronin
Tom Crown
Reine Dahlqvist
Peter Dawson
Robert Dawson
Allan Dean
Bruce Dickey
Richard Diehl
Lynn Dillon
Steve Dillon
Mark Dodson
Patrick Dougherty
Sherwood Dudley
Ralph Dudgeon
Richard Dundas
Bernard Ebbinghouse
G. Norman Eddy
Hugh Eddy
David Edwards
Michael Edwards
A. Egger

A. Egger

Thomas Ekman

Lyn Elder

Robert E. Eliason

Randolph D. Emerick

Gerald Endsley

Kerry England

John Ericson

Stephen Escher

Thomas Everett

Stephen Faraudo

Susan Farrell
Joan Feigenbaum
Henry Fischer
Joseph Fierce
Thomas Freas
Arnold Fromme
Richard Fuller
Jack Gallagher
Chris Gekker
Haags Gemeentemuseum
Richard George
Vince Giordano
John Givre
Steve Glover
Brian Goff
Robert Goodman
Charles Gouse
Stentiford Graeme
R. Luce Denis
de Granddidier
Robert Grechesky
Lorenzo Greenwich, Jr.
Ernest "Chip" Gross
Marcia Grumme
David Guion
Karl Hachenberg
Karen Hansen
Ben Harms
Vernon Harp Jr.
Judith W. Harrington
Michael Hathaway
Robert Hazen
Douglas Hedwig
Mark Henson
Trevor Herbert
Flora Herriman
John Hicks
Tom Hiebert
Tom




\begin{tabular}{|c|c|c|}
\hline D.J. Hildebrandt & David Lasocki & Philip Palmer \\
\hline John Hildebrand & Frank M. Legate & J.S. Patterson \\
\hline Jay Hilfiger & Philip Levin & Arthur Pecht \\
\hline Marta Hofacre & H.M. Lewis Jr. & Ben Peck \\
\hline David Hogan Smith & Bonnie Lindblom & Rinaldo Pellizzari \\
\hline Fred Holmgren & Arthur Linser & Hans C. Peterson \\
\hline Chatherine homo-Lechner & Royce Lumpkin & William A. Pfund \\
\hline Thomas Hooper & Steven Lundahl & Peter Piacquadio \\
\hline Jerry G. Horne & George Lyman & Fordyce C. Pier \\
\hline Valerie Horst & Jim MacDougall & Terry Pierce \\
\hline John Horton & Michael Malloy & Andrew Pinnock \\
\hline Brian Howard & Loren Marsteller & S.E. Plank \\
\hline Wayne Huber & Ray Mase & Fritz Pohl \\
\hline Amy Hudson & Roger Mastalir & Keith Polk \\
\hline Charles J. Hudson & Bill Mathews & Alexander Pollock \\
\hline T.D. Hughston & Victor Mattfield & Larry Price \\
\hline J.B. Hunter & Gus Mazzocca & Jeffrey Quick \\
\hline Larry Hutchings & John R. McCann & Samuel D. Quigley \\
\hline Institute of Popular Music & Rebecca McClone & Bruce Randall \\
\hline for Mediterranean Europe & Thomas Meacham & James Rhoades \\
\hline Andy Isca & Henry Meredith & Robert Rieder \\
\hline Sharon Jacobson & Paul Merrill & Trevor Robinson \\
\hline Larry Johansen & Frank Mesich & John Rogers \\
\hline Michael Johns & Mark A. Metzler & William Rogers \\
\hline Stephen Johns & Steven Moise & Jesse Rosenberg \\
\hline Craig Johnson & Andras Molnar & Viola Roth \\
\hline Mark R. Jones & Vincent Monaco & Douglas Russell \\
\hline Robert Jones & Christopher Monk & David Rycroft \\
\hline Stephen Jones & Jeremy Montagu & William Scarlett \\
\hline Debra Kahler & Donald Montalto & Gerhard Schmeltekopf \\
\hline Donald Kahn & Robert Montesi & Marcio Selles \\
\hline Lyn Kamowitz & Christiane Munkwitz & Rick Seraphinoff \\
\hline Bruno Kampmann & Michael Munkwitz & Steve Silverstein \\
\hline Didier Kanmacher & Floyd Munson & Aaron Smith \\
\hline Karl Kaub & Arnold Myers & David Hogan Smith \\
\hline McDowell Kenley & Michael Myers & Jack Smith \\
\hline Doug Kirk & Gary Nagels & William Smith III \\
\hline Jeffrey Kite-Powell & Michael Nagy & Don Smithers \\
\hline Linda Klein & Nigel Nathan & Jeffrey Snediker \\
\hline Walter Knapp & Hollister Nelson & Karen Snowberg \\
\hline Joel Kramme & Milton Nelson & Richard Sorenson \\
\hline Craig Kridel & Ron Nelson & Scott Sorenson \\
\hline Jay Krush & Glen Newton & Frederick Starr \\
\hline J. Quentin Kuyper & Richard Norment III & Nelson Starr \\
\hline Barbara Lambert & Jeff Nussbaum & Crispian Steele-Perkins \\
\hline G.B. Lane & Connie Palmer & Manny Stevens \\
\hline
\end{tabular}


Robb Stewart

Robert Stibler

Daniel Stillman

John Stork

Streitwieser Foundation

Orum Stringer

Wolfgang Suppar

Edward Tarr

Scott Temple

James TenBensel

George Theokritoff

John Charles Thomas

Caldwell Titcomb

Francis James Tomes

Tom Treas
Juan Ramon Ullibarri Galparsoro

Timothy Urban

Joe R. Utley

Geert Jan Van der Heide

Richard Van Hessel

David Vining

Robert Wagenknecht

John Wall

Richard Wallingford

James Waters

John Wates

Alan S. Weakley

John Webb

Larry Weed

Russell Weisman
James West

James Wheat

Sandra Wheat

Clifford Wheeler

Chris Whitehead

Leon Whitsell, M.D.

Scott Wilkinson

Carl Willets

Frederick Williams

Dan Woolpert

David Workman

Douglas Yeo

Tom Yoder

Tom Zajac

Leonard Zon

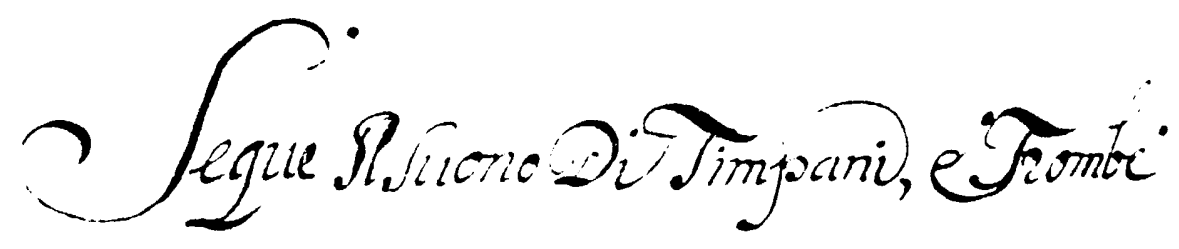

